When using detergents for cleaning beaches, it has been shown very clearly that it is most important to utilise the natural mixing effect of the sea. Detergent application across the beach parallel to the incoming tide should be timed so that the breaking wave action mixes the oil detergent - sand certainly within one hour of application, and the sooner the better.

GuNKEL: What is the stability of the emulsion formed under these conditions?

BROCKIS: I cannot give a specific answer to that. However, for practical purposes it is sufficient to say that application in this way is very effective. The mixing principle must also be applied when cleaning rocks. Where wave-breaking action will not cover soiled rocks, after applying detergents, it is necessary to direct a high pressure jet of water into the treated areas.

SchöвERL: What kind of emulsifiers were used in Cornwall?

Brockis: Several brands were used. As far as is known, most if not all of them were the non-ionic variety. It is believed that the majority were based on polyalkylene oxide-alkyl phenol condensates.

\title{
Toxizität von Mineralölemulgatoren auf See- und Süßwasserorganismen
}

KüHL: Die Mittel, die in Cuxhaven geprüft wurden, erhielt ich von Herrn Oberbaurat Reuter. Es waren dies: Oil-Ex, Anti-Oil, TS 5, Sillarit, Vecom B 24, PS 777, Moltoclar, J 502, Gamlen, Elimax, Slix, Peroclean. Als Testtiere dienten: die Larven von Spioniden, Balaniden und Schnecken; ferner Copepoden, Mysideen, Amphipoden, einige andere Plankter und schließlich Garnelen, Strandkrabben und Jungschollen (O-Gruppe). Die Versuche wurden im Cuxhavener Brackwasser (15 bzw. 20\% Salzgehalt) und Helgoländer Wasser (32\% Salzgehalt) durchgeführt. Die Letalitätsgrenzen lagen zwischen $2 \times 10^{-4}$ und $10^{-5}$ Verdünnung. Bei Mischungen mit Ol lagen die Grenzen in den gleichen Größenordnungen wie bei den reinen Emulgatoren. Die Versuche wurden beendet, wenn nach 6 Tagen keine sichtbare Schädigung mehr festzustellen war. Die Versuchstiere wurden dann in reines Seewasser zurückgesetzt. Bei den Bekämpfungen von Ölverschmutzungen durch Emulgatoren sind die Schadwirkungen im wesentlichen auf die Emulgatoren und in weitaus geringerem Maße auf das OI als solches zurückzuführen (vgl. KüHL \& MANN 1967).

MANN: Ole können nicht nur toxisch wirken, sondern haben auch noch andere unangenehme Eigenschaften, nämlich die Beeinflussung des Geschmackes von Fischen. Bekanntlich sind Elbfische vielfadh ungenießbar, wenn sie mit Olverschmutzungen in Berührung gekommen sind. Dies ist zum Beispiel vor Jahren bei Maifischen im Raum von Cuxhaven der Fall gewesen, auch von Plattfischen kennt man ähnliches. In diesem Zusammenhang sei darauf hingewiesen, daß auch Phenole den Fischen einen unangenehmen Beigeschmack beibringen können. Bekannt ist dies aus Gewässern, die durch phenolhaltige Abwässer verschmutzt wurden. Es ist daran zu denken, daß bei Vorhandensein von Emulgatoren im Wasser die Geschmacksbeeinflussung der Fische durch Ole und Phenole verstärkt werden kann, wie es von den Tensiden her bekannt ist. Hinzu kommt noch, daß nicht nur durch direkten Kontakt der Fische mit Ol und Olemulsionen der unangenehme Geschmack erzielt werden kann, sondern auch auf dem Wege über die Nährtiere. Werden zum Beispiel Forellen mit "schlecht" schmeckenden Fischen gefüttert, so nehmen die Forellen sehr schnell durch Speicherung der Geschmacksstoffe im Fett den "schlechten" Geschmack an. Auch die Eier von Geflügel, das mit beanstandeten Fischen gefüttert wurde, schmeckten nach Ölen und Phenolen (MaNN 1960). In Binnengewässern wurden schon neben Emulgatoren Bindemittel zur Bekämpfung von Olverschmutzungen mit Erfolg eingesetzt. Diese Mittel, bei denen es sich um Stoffe auf der Basis von Holzmehl, Torf, Kunststoff oder Gesteinsglas handelt, sind fischereilich unbedenklich (MANN 1966).

WallhäUsser: Haben Sie die Toxizität geprüft, wenn Sie beispielsweise an Kieselgur Ol absorbieren, und das sinkt ab? Geht das Ol von der Oberfläche des Kieselgurpartikels wieder ab?

ManN: Bei den Ölbindern muß man zwei Typen unterscheiden. Die eine Gruppe, die ich oben erwähnte, bleibt auch nach Aufnahme des OOls schwimmfähig und kann von der Oberfläche 
abgeschöptt werden. Eine andere Gruppe, zum Beispiel „Oilsink“, sinkt nach der Olaufnahme zu Boden. Diese Stoffe sind abzulehnen. Wenn sie absinken, decken sie den Boden ab und behindern den Stoffaustausch zwischen Boden und Wasser. Auch ist bekannt, daß sie nach einiger Zeit das aufgenommene $O \mathrm{O}$ an das umgebende Wasser wieder abgeben können.

Wal.reäusser: Wird nicht diese Kontaktschicht der Sedimente mit dem abgesunkenen Ol sehr schnell wieder durch neue Sedimente zugedeckt? Wie wirkt es sich dann aus?

Gunket: Ein Überdecken kann normalerweise nur am Strand auftreten. In der freien See ist die Zuwachsrate der Sedimente viel zu gering, um hier irgendeinen. Einfluß zu haben.

MANN: In diesem Zusammenhang sei darauf hingewiesen, daß auch manche Ole am Boden verharzen und dann eine Besiedlung mit Organismen unmöglich machen.

HELLMANN: Ich darf aber dazu noch einmal sagen, daß Absinkmittel immer wieder angeboten werden. Wir haben uns in der Bundesanstalt von Zeit zu Zeit mit ihnen zu befassen. Viele hiervon sind nach geheimgehaltenen Patentrezepten hergestellt worden.

\section{Ollabbau durch Rein-, Anreicherungs- und Mischkulturen von Bakterien}

SCHÖBERL: STEWART et al. (1960) haben, soweit mir bekannt ist, lediglich mit einem Bakterienstamm gearbeitet. Sie haben nicht behauptet, daß Paraffine ganz allgemein nur bis zu einem Ester abgebaut würden, sondern sie haben gefunden, daß Alkane mit mehr als $10 \mathrm{C}$-Atomen von dem von ihnen verwendeten Micrococcus-Stamm abgebaut werden. Dabei tritt eine Esteranreicherung in der Kulturlösung auf. Unzählige Arbeiten auf diesem Gebiet haben gezeigt, daß Kohlenwasserstoffe mit weniger als $10 \mathrm{C}$-Atomen bis zu $\mathrm{CO}_{2}$ und $\mathrm{H}_{2} \mathrm{O}$ abgebaut werden.

Schwersfurth: Wie Herr RÜBELT ausführte, haben wir begonnen, uns mit dem Abbau von Kohlenwasserstoffen zu beschäftigen. Für die ersten Versuche mit kohlenwasserstoffabbauenden Mikroorganismen, die den Zweck haben, unseren Chemikern Ausgangsmaterial für organischchemische Gruppenbestimmungen zu liefern und verschiedene Stämme zu isolieren, verwenden wir vorerst Glasröhren, in die unten eine Fritte eingeschmolzen ist. Die Kolben fassen 11 mineralische Nährlösung, die 2 bis $4 \mathrm{ml}$ Kohlenwasserstoff ( $\mathrm{C}_{16}$, Heizöl, Rohöl) erhalten, das auf die Oberfläche gegeben wird. Die Primärkulturen werden mit ölverunreinigtem Boden beimpft. Nach 2 bis 3 Tagen der Belüftung und bei Zimmertemperatur beginnt die Emulgierung der Kohlenwasserstoffe, die jedoch nicht einsetzt, wenn mit Stickstoff begast wird. Die sich entwickelnden Bakterienpopulationen zeigen nicht nur eine Abhängigkeit vom Kohlenwasserstoff, sondern auch vom Alter der Kulturen, woraus gefolgert wird, daß durch den ersten Angriff auf die Kohlenwasserstoffe Stoffwechselprodukte entstehen, die von anderen Bakterienarten in der Folge verwertet werden können. Das Arbeiten mit Mischkulturen hat den Vorteil, daß man hier mit gezielten Isolierungen für spätere Versuche beginnen kann. Limitierende Faktoren jeglichen Abbaues von C-Verbindungen sind - wie wir es auch experimentell im Süßwasser fanden - anorganische N-Quellen und der $\mathrm{PO}_{4}$-Gehalt der Lösungen. Da sich auf die beim Kohlenwasserstoffabbau gebildeten Zwischenprodukte unsere Aufmerksamkeit besonders richten wird, versuchen wir auch, beispielsweise Rohöl, das von der ersten Bakterienpopulation angegriffen wurde, von weiteren Organismengruppen weiter abbauen zu lassen. Mit kontinuierlichen Kulturen kann man hier offensichtlich gut arbeiten. Untersuchungen an Reinkulturen sind zwar später unerläßlich, doch bei dem Versuch, Stoff wechselprodukte chemisch-analytisch zu untersuchen, die nach Olverunreinigungen von Boden und Grundwasser in diesem transportiert werden können, muß Wert auf möglichst konstante Mischpopulationen gelegt werden, um wenigstens einen kleinen Teil der Faktoren des natürlichen Milieus zu imitieren.

SснӧвеRL: Es ist richtig, daß man in der Natur Mischkulturen vorliegen hat. Aber wenn Sie den Versuch unternehmen wollen, Zwischenprodukte des Kohlenwasserstoffabbaus zu identi- 